\title{
Flujos de capital externo y crecimiento económico múltiple
}

\author{
Enrique R. Casares*
}

\section{RESUMEN}

Se desarrolla un modelo de crecimiento endógeno con dos sectores: comerciable (manufacturero) y no comerciable (no manufacturero). El conocimiento tecnológico es producido únicamente en el sector comerciable. El sector no comerciable puede usar este conocimiento. Se estudia cómo la economía responde a una mayor movilidad del capital. Se muestra que la entrada de capital externo produce una apreciación del tipo de cambio real. Así, el sector comerciable (líder en conocimiento tecnológico) es perjudicado por la entrada de capital. Sin embargo, dado que existen rendimientos crecientes a escala, la tasa de crecimiento de la economía puede disminuir o aumentar, con posibilidad de equilibrio múltiple, en el largo plazo.

Palabras clave: sector comerciable, aprendizaje, movilidad del capital, tipo de cambio real, crecimiento.

Clasificación JEL: F21, F36, F43, O41.

\begin{abstract}
We develop an endogenous growth model with two sectors, tradable (manufacturing) and non-tradable (non-manufacturing). Technological knowledge is only produced in the tradable sector. The non-tradable sector can use this knowledge. We study how the economy responds to an increase in capital mobility. We show that the inflow of foreign capital produces an appreciation of the real exchange rate. Thus, the tradable sector (leader in technological knowledge) is damaged by the capital inflow. However, given that there are increasing returns to scale, the economy growth rate can decrease or increase, with possibility of multiple equilibrium, in the long run.
\end{abstract}

Key words: tradable sector, learning, capital mobility, real exchange rate, growth.

Classification JEL: F21, F36, F43, O41.

\footnotetext{
* Doctor en economía por The University of London. Profesor-investigador en el Departamento de Economía de la Universidad Autonoma Metropolitana-Azcapozalco: ercg@correo.azc.uam.mx
} 


\section{INTRODUCCIÓN}

La premisa sobre la apertura financiera es que la entrada de capital externo a los países en desarrollo producirá una reducción en el costo del capital, un aumento en la inversión y la producción (véase Henry, 2003). Sin embargo, Prasad, Rajan y Subramanian (2007) han mostrado que economías en desarrollo con saldos positivos de la cuenta corriente tuvieron tasas de crecimiento mayores que economías con saldos negativos para el periodo 1970-2000. Por tanto, las economías que dependieron menos del capital externo crecieron más. Igualmente, Aizenman, Pinto y Radziwill (2007) muestran que las economías en desarrollo con proporciones mayores de autofinanciamiento (proporción del capital doméstico financiado con ahorro doméstico) crecieron más que las economías con proporciones de autofinanciamiento menores para los años 1990-1999. Finalmente, en un amplio resumen de la bibliografía, Obstfeld (2008) concluye que es difícil encontrar evidencia macroeconómica inequívoca que muestre que la apertura financiera mejora el funcionamiento de las economías (véase también Kose, Prasad, Rogoff y Wei, 2006).

Los resultados de una liberalización financiera dependerán de las características de cada país (véase Prasad, Rajan y Subramanian, 2006). Así, algunos autores han argumentado que la entrada de capital externo puede conducir a una apreciación del tipo de cambio real y esto, a su vez, dañar la rentabilidad del sector manufacturero. Si el sector manufacturero es uno de los motores básicos de crecimiento de las economías en desarrollo, la apreciación del tipo de cambio real podría dañar el crecimiento agregado de la economía. En Rodrik (2008) se muestra que un tipo de cambio real subvaluado estimuló el crecimiento económico en países en desarrollo en los años 1950-2004. Asimismo, D. Rodrik menciona que una posible explicación de esta causalidad es la existencia de externalidades de aprendizaje en el sector comerciable o manufacturero (véase también P. Gala, 2008).

En este artículo se estudia el impacto de una mayor movilidad del capital externo en el tipo de cambio real y el crecimiento económico en una pequeña economía abierta. Por tanto, se desarrolla un modelo de crecimiento endógeno con dos sectores: comerciable y no comerciable. Se puede interpretar al sector comerciable como el sector manufacturero y al sector no comerciable como el sector no manufacturero (construcción y servicios). El sector comerciable es la fuente del conocimiento tecnológico, el cual es utilizado en el sector del bien no comerciable. Así, en el modelo hay dos externalidades de aprendizaje. 
El modelo se sitúa en la bibliografía de macroeconomía internacional que divide la economía en un bien comerciable y en un bien no comerciable. En modelos con acumulación de capital, Obstfeld (1989) supone que el bien comerciable puede ser transformado en capital y Turnovsky (1991) considera que el bien de inversión es no comerciable. Brock y Turnovsky (1994) desarrollan un modelo con dos tipos de capital: uno comerciable (equipos) y otro no comerciable (estructuras) y Turnovsky (1996) presenta un modelo de crecimiento endógeno, en donde el capital físico es comerciable y el capital humano es no comerciable (para un modelo semejante, Neto, 2009, analiza el efecto de la movilidad del capital en la velocidad de convergencia). Para estudiar la enfermedad holandesa, es decir, el resultado adverso sobre el sector comerciable de un descubrimiento de un recurso natural, Torvik (2001) presenta un modelo en donde ambos sectores contribuyen al aprendizaje por la práctica y hay desbordamientos de los conocimientos entre los sectores. El modelo desarrollado en este artículo es diferente de los existentes.

La economía posee deuda externa y se supone que los inversionistas externos perciben un riesgo de no pago de la deuda externa. En consecuencia, se introduce un parámetro que mide la prima de riesgo-país sobre la tasa de interés internacional. Suponiendo una forma de paridad de tasas de interés ajustado con riesgo, tenemos que la tasa de interés o el costo sobre el capital doméstico y la deuda externa es igual a la tasa de interés mundial más la prima de riesgo-país. El parámetro de riesgo mide el grado de movilidad del capital externo. Así, cuando el parámetro disminuye, la movilidad del capital aumenta, es decir, a menor riesgo-país, la oferta de fondos externos aumenta y se producirá una entrada de capital externo a la economía.

En el estado estacionario se analiza cómo la economía responde a una mayor movilidad del capital externo. Se muestra analíticamente que un aumento en el capital externo produce un aumento en el precio relativo del bien no comerciable, es decir, el tipo de cambio real se aprecia. Esta apreciación causa que el sector no comerciable atraiga recursos a costa del sector comerciable (manufacturero). Así, en el sector comerciable la fracción del trabajo empleado disminuye y la acumulación de capital se desalienta. Por tanto, el modelo siempre predice que el sector comerciable -el sector líder en términos tecnológicos- es perjudicado por la entrada de capital externo. Sin embargo, dado que existen rendimientos crecientes a escala, la tasa de crecimiento de la economía puede disminuir o aumentar en el largo plazo, dependiendo de las características de cada economía. Asimismo, el nivel de deuda externa y la tasa de crecimiento de la economía pueden mostrar equilibrio múltiple. Por ello, el modelo refleja la bibliografía empírica antes mencionada. 
En particular, como señalan Urrutia y Meza (2010), durante la liberalización de la economía mexicana y la entrada de capitales asociada, la tasa de interés para préstamos a México (tasa de interés real de los bonos del Tesoro a tres meses más el diferencial J. P. Morgan de los bonos Brady) cayó de 15\% en 1990 a 5\% en 1994, con un aumento súbito durante la crisis de 1995 para bajar a menos de 5\% en 2002. Mientras, el índice del tipo de cambio real multilateral del peso mexicano pasó de 104.5 en diciembre de 1988 a 68.1 en enero de 1994, apreciándose $36 \%$ (una disminución del índice representa una apreciación, Banco de México). Debido a la crisis de 1995, el tipo de cambio real multilateral se depreció bruscamente, para continuar con su apreciación, bajando el índice a 64.9 en diciembre de 2002. Así, la apreciación para el periodo 1988-2002 fue de 39\%. Utilizando el tipo de cambio bilateral México-Estados Unidos (el multilateral tiene un comportamiento similar), Urrutia y Meza (2010) encuentran que esta apreciación del tipo de cambio real en el periodo 1988-2002 se explica principalmente por el efecto Balassa-Samuelson (un crecimiento de la productividad más alta en el sector comerciable comparado con el no comerciable) y por la disminución de la tasa de interés. Así, la apreciación del tipo de cambio real del peso mexicano, producido en parte por la liberalización financiera, redujo la rentabilidad de exportar. El resultado final fue un crecimiento deficiente de la economía mexicana, en parte por la apreciación y en parte por otros factores, con una tasa de crecimiento promedio del ingreso per cápita en dólares internacionales de $1.6 \%$ anual para el periodo $1988-2002$.

El artículo está organizado de la siguiente manera: En la sección I se desarrolla un modelo de crecimiento endógeno de una pequeña economía abierta. En la sección II se redefine el modelo en términos de variables estacionarias. En la sección III se presenta el sistema dinámico. En la sección IV se estudia el estado estacionario y la dinámica de transición. Al final, se presentan las conclusiones.

\section{LA ECONOMÍA}

La economía es una pequeña economía abierta con comercio de bienes y activos. Por tanto, la economía toma como dados el precio del bien comerciable y la tasa de interés mundial. Se supone que los dos bienes, comerciable y no comerciable, son producidos, consumidos y acumulados. El bien en cada sector es producido por medio de capital físico, trabajo y conocimiento tecnológico. Por simplicidad, el sector comerciable es el único sector que genera conocimiento tecnológico por medio de un aprendizaje en la práctica (learning by doing). El conocimiento pro- 
ducido en el sector comerciable puede ser utilizado en el sector no comerciable. El trabajo es homogéneo y se mueve libremente entre los dos sectores. La oferta total de trabajo es constante. El gasto total en consumo es una fracción constante del ingreso disponible.

\section{Las empresas}

Se supone que la función de producción del sector comerciable es Cobb-Douglas:

$$
Y_{T}=A_{T} K_{T}^{\alpha} L_{T}^{1-\alpha} E_{1}
$$

donde $Y_{T}$ es la producción del bien comerciable, $A_{T}$ es un parámetro productivo, $K_{T}$ es el acervo de capital físico acumulado del bien comerciable, $L_{T}$ es el trabajo empleado en el sector, $\alpha$ y $1-\alpha$ son las participaciones de $K_{T}$ y $L_{T}$, respectivamente, y $E_{l}$ es una externalidad de aprendizaje. Se supone que $K_{T}$ es usado únicamente en el sector comerciable.

El conocimiento tecnológico es creado a partir de un aprendizaje en la práctica en el sector (learning by doing). Así, el conocimiento es un subproducto de la inversión en el sector. Por tanto, $E_{l}$ es el efecto externo de $K_{T}$ en la función de producción del sector comerciable. Para generar crecimiento endógeno, se supone que $E_{1}=K_{T}^{1-\alpha}$; así, la función de producción del sector comerciable tiene rendimientos constantes en el capital ampliamente medido (véase Romer, 1989).

Se define $P_{T}^{*}$ como el precio constante mundial del bien comerciable. Se usa el bien comerciable como numerario $\left(P_{T}^{*}=1\right)$. Además, se define a $r^{*}$ como la tasa de interés mundial que es constante. Se introduce un parámetro $\xi$, que mide la prima de riesgo-país sobre $r^{*}$. Eicher y Hull (2004) definen a $\xi$ como una función creciente de la relación deuda-capital (véase también Eicher y Turnovsky, 1999). En este artículo, para simplificar, el parámetro $\xi$ es exógeno. Suponiendo una forma de paridad de tasas de interés ajustado con riesgo, tenemos que la tasa de interés o el costo sobre el capital doméstico y la deuda externa es igual a $r^{*}+\xi$. Se supone que el parámetro $\xi$ mide el grado de movilidad del capital externo. Así, cuando el parámetro disminuye, aumenta la movilidad del capital, es decir, a menor riesgo-país, la oferta de fondos externos aumenta y se producirá una entrada de capital externo a la economía. El costo de $K_{T}$ es $R_{T}=\left(r^{*}+\xi+\delta_{K T}-\dot{P}_{T}^{*} / P_{T}^{*}\right)$. Dado que $P_{T}^{*}$ es el numerario y que $K_{T}$ tiene una tasa de depreciación cero $\left(\delta_{K T}=0\right)$, el costo de $K_{T}$ es $R_{T}=r^{*}+\xi$. Por tanto, el nivel de $R_{T}$ está fijado por la tasa de interés mundial más $\xi$. Las empresas en el sector comerciable maximizan 
beneficios tomando la externalidad como dada. Las condiciones de primer orden son:

$$
\begin{aligned}
& w_{T}=A_{T} K_{T}(1-\alpha) L_{T}^{-\alpha} \\
& R_{T}=r^{*}+\xi=A_{T} \alpha L_{T}^{1-\alpha} .
\end{aligned}
$$

La ecuación (2) dice que el salario es igual al valor del producto marginal del trabajo en el sector comerciable. La ecuación (3) indica que el costo de $K_{T}$ es igual al producto marginal de $K_{T}$. Por tanto, $r^{*}+\xi$ es la tasa de interés sobre los activos domésticos.

Respecto al sector no comerciable, la función de producción es CobbDouglas:

$$
Y_{N}=A_{N} K_{N}^{\beta} L_{N}^{1-\beta} E_{2}
$$

donde $Y_{N}$ es la producción del bien no comerciable, $A_{N}$ es un parámetro productivo, $K_{N}$ es el acervo de capital físico acumulado con el bien no comerciable, $L_{N}$ es el trabajo empleado en el sector, $\beta$ y $1-\beta$ son las participaciones de $K_{N}$ y $L_{N}$, respectivamente, y $E_{2}$ es una externalidad. El acervo de $K_{N}$ es usado únicamente en el sector no comerciable.

Existe un efecto de desbordamiento del conocimiento entre los sectores. Así, $E_{2}$ es la contribución del conocimiento tecnológico (generado en el sector comerciable) en la producción de bienes no comerciables. Además, para generar crecimiento endógeno, se supone que $E_{2}=K_{T}^{1-\beta}$; así la función de producción del sector no comerciable tiene rendimientos constantes en el capital ampliamente medido.

Se define $p_{N}$ como el precio relativo del bien no comerciable en términos del bien comerciable. El costo de $K_{N}$ es $R_{N}=\left(r^{*}+\xi+\delta_{K N}-\dot{p}_{N} / p_{N}\right)$. Dado que $K_{N}$ tiene una tasa de depreciación cero $\left(\delta_{K N}=0\right)$, la renta de $K_{N}$ es $R_{N}=\left(r^{*}+\xi-\dot{p}_{N} / p_{N}\right)$, donde $\dot{p}_{N} / p_{N}$ es la tasa de crecimiento de $p_{N}$ o las ganancias de capital. Las empresas no comerciables maximizan beneficios tomando la externalidad como dada. Las condiciones de primer orden son:

$$
\begin{gathered}
w_{N}=p_{N} A_{N} K_{N}^{\beta} K_{T}^{1-\beta}(1-\beta) L_{N}^{-\beta} \\
R_{N}=r^{*}+\xi-\frac{\dot{p}_{N}}{p_{N}}=A_{N} \beta K_{N}{ }^{\beta-1} K_{T}^{1-\beta} L_{N}^{1-\beta} .
\end{gathered}
$$

La ecuación (5) dice que el salario es igual al valor del producto marginal del trabajo en el sector no comerciable. La ecuación (6) es la condición de 
equilibrio dinámica para $K_{N}$. Así, la ecuación dice que el costo de $K_{N}$ es igual al producto marginal de $K_{N}$.

Es importante señalar que en un contexto agregado, el tipo de cambio real está definido como el nivel de precios del país extranjero (en moneda nacional) dividido entre el nivel de precios del país doméstico. En un contexto desagregado de bienes comerciables y no comerciables, el tipo de cambio real está definido como el valor ponderado del nivel de precios relativos de los bienes no comerciables del país extranjero (tipo de cambio nominal igual a uno) dividido entre el nivel de precios relativos de los bienes no comerciables del país doméstico. Considerando el nivel de precios relativos del país extranjero como dado, el tipo de cambio real está inversamente relacionado con el nivel de precios relativos de los bienes no comerciables del país doméstico. Por tanto, un aumento en $p_{N}$ conducirá a una apreciación del tipo de cambio real.

\section{Los individuos}

La restricción de los individuos es:

$$
\begin{aligned}
\dot{D} & =\left(r^{*}+\xi\right) D+C_{T}+p_{N} C_{N} \\
& +I_{T}+p_{N} I_{N}-Y_{T}-p_{N} Y_{N},
\end{aligned}
$$

donde $D$ es la deuda externa, $\dot{D}$ es el incremento en el tiempo de la deuda externa $\left(r^{*}+\xi\right) D$ es el pago de intereses; así $r^{*}+\xi$ es la tasa de interés sobre la deuda externa, el término $C_{T}$ es el consumo en el bien comerciable, $C_{N}$ es el consumo en el bien no comerciable, $I_{T}$ es la inversión en $K_{T}, I_{N}$ es la inversión en $K_{N}$, y $Y_{T}+p_{N} Y_{N}=Y$ es la producción total o ingreso total. La ecuación (7) está expresada desde el punto de vista de un individuo deudor; así el pago de interés más el gasto total.

Por simplicidad, no hay elección intertemporal. Por tanto, se supone que el gasto total en consumo es una fracción constante del ingreso disponible:

$$
p_{C} C=\hat{c}\left[Y_{T}+p_{N} Y_{N}-\left(r^{*}+\xi\right) D\right],
$$

donde $p_{C}$ es el índice de precios relativos al consumidor, $C$ es el consumo agregado real y $\hat{c}$ es la propensión a consumir, que es constante $(0<\hat{c}<1)$.

Una vez que se tiene el gasto total en consumo, se determina la composición de la canasta de consumo. Así, se supone que las demandas de consumo 
para el bien comerciable y para el bien no comerciable resultan de la maximización estática de la función de utilidad:

$$
u=B C_{T}^{\gamma} C_{N}^{1-\gamma}
$$

sujeto al gasto total en consumo $p_{C} C=C_{T}+p_{N} C_{N}$, donde $B=1 /\left[\gamma^{\gamma}(1-\gamma)^{1-\gamma}\right]$ es un parámetro, $\gamma$ y $1-\gamma$ son las participaciones de $C_{T}$ y $C_{N}$ en relación con el gasto total en consumo, respectivamente. El índice de precios relativos al consumidor puede ser definido como $p_{C}=p_{N}^{1-\gamma}$. Entonces, se obtiene:

$$
\begin{aligned}
C_{T} & =\gamma p_{C} C \\
C_{N} & =(1-\gamma) \frac{p_{C} C}{p_{N}},
\end{aligned}
$$

en donde la ecuación (10) es la demanda de $C_{T}$ y la ecuación (11) es la demanda $\operatorname{de} C_{N}$.

\section{Mercados}

Dado que el precio relativo del bien no comerciable es flexible, la oferta del bien no comerciable siempre es igual a la demanda. Por tanto, la condición de equilibrio para el mercado del bien no comerciable es:

$$
p_{N} Y_{N}=p_{N} C_{N}+p_{N} I_{N}
$$

en donde $I_{N}=\dot{K}_{N}$. Con las ecuaciones (7) y (12) se obtiene la condición de equilibrio para el mercado del bien comerciable:

$$
\dot{D}=\left(r^{*}+\xi\right) D+C_{T}+I_{T}-Y_{T}
$$

en donde $I_{T}=\dot{K}_{T}$. La cuenta corriente deficitaria es:

$$
\dot{D}=\left(r^{*}+\xi\right) D-N X
$$

en donde $N X$ es el saldo comercial. Con las ecuaciones (13) y (14) se puede reescribir la condición de equilibrio para el mercado del bien comerciable como: 


$$
Y_{T}=C_{T}+I_{T}+N X
$$

La ecuación (15) muestra una condición de equilibrio para un mercado con flujo internacional en bienes.

\section{EL MODELO EN VARIABLES ESTACIONARIAS}

Dado que las variables $K_{T}$ y $K_{N}$ crecerán permanentemente, es necesario definir las variables del modelo como variables estacionarias, es decir, variables que sean constantes en el estado estacionario (véase Barro y Sala-i-Martin, 1995). Así, se define a $z=K_{N} / K_{T}$ como la primera variable estacionaria. Además, se supone que la oferta total de trabajo, $L$, es constante y normalizada a 1 . De este modo, la condición de equilibrio en el mercado laboral es: $L_{T}+L_{N}=L=n+$ $(1-n)=1$, donde $n$ es la fracción del trabajo empleado en el sector comerciable y $(1-n)$ es la fracción del trabajo empleado en el sector no comerciable. Como $n$ es constante en el estado estacionario, la variable $n$ es la segunda variable estacionaria. Se define a $d=D / K_{T}$ como la tercera variable estacionaria. Asimismo, como $p_{N}$ debe ser constante en el estado estacionario, $p_{N}$ es la cuarta variable estacionaria.

Por tanto, la función de producción del sector comerciable en términos de variables estacionarias es:

$$
Y_{T}=A_{T} K_{T} n^{1-\alpha}
$$

y las condiciones marginales para el sector comerciable en términos de variables estacionarias son:

$$
\begin{gathered}
w_{T}=A_{T} K_{T}(1-\alpha) n^{-\alpha} \\
r^{*}+\xi=A_{T} \alpha n^{1-\alpha} .
\end{gathered}
$$

Asimismo, la función de producción del sector no comerciable en términos de variables estacionarias es:

$$
Y_{N}=A_{N} K_{T} z^{\beta}(1-n)^{1-\beta}
$$

y las condiciones marginales para el sector no comerciable en términos de variables estacionarias son: 


$$
\begin{gathered}
w_{N}=p_{N} A_{N} z^{\beta} K_{T}(1-\beta)(1-n)^{-\beta} \\
r^{*}+\xi-\frac{\dot{p}_{N}}{p_{N}}=A_{N} \beta \mathrm{z}^{\beta-1}(1-n)^{1-\beta} .
\end{gathered}
$$

Se supone que $\alpha>\beta$; así el sector comerciable es más intensivo en capital que el sector no comerciable. Turnovsky (1997) analiza las consecuencias de este tipo de supuesto.

Con las ecuaciones (17) y (20) se obtiene la condición estática de asignación eficiente del trabajo entre los dos sectores $\left(w_{T}=w_{N}\right)$. Así, la condición es:

$$
A_{T}(1-\alpha) n^{-\alpha}=p_{N} A_{N} z^{\beta}(1-\beta)(1-n)^{-\beta}
$$

Esta condición dice que el valor del producto marginal del trabajo en ambos sectores debe ser igual. La condición de equilibrio del mercado del bien comerciable en términos de variables estacionarias, ecuación (13), es:

$$
\dot{d}=\left(r^{*}+\xi\right) d+\frac{C_{T}}{K_{T}}+(1-d) \frac{\dot{K}_{T}}{K_{T}}-A_{T} n^{1-\alpha},
$$

donde $\dot{K}_{T} / K_{T}=g_{T}$ es la tasa de crecimiento de $K_{T}$. Usando las ecuaciones (8), (16) y (19) se obtiene el gasto total en consumo en términos de variables estacionarias:

$$
p_{C} C=\hat{c}\left[A_{T} K_{T} n^{1-\alpha}+p_{N} A_{N} K_{T} z^{\beta}(1-n)^{1-\beta}-\left(r^{*}+\xi\right) D\right]
$$

Por tanto, usando las ecuaciones (10) y (24), el valor de $C_{T} / K_{T}$ en la ecuación (23) es:

$$
\frac{C_{T}}{K_{T}}=\gamma \hat{c}\left[A_{T} n^{1-\alpha}+p_{N} A_{N} z^{\beta}(1-n)^{1-\beta}-\left(r^{*}+\xi\right) d\right] .
$$

Para obtener la tasa de crecimiento de $K_{N}$, se usa la condición de equilibrio para el mercado del bien no comerciable, ecuación (12), con la función de producción del sector no comerciable, ecuación (19), el nivel de $C_{N}$, ecuación (11), y la identidad $I_{N}=\dot{K}_{N}$; se obtiene:

$$
\frac{\dot{K}_{N}}{K_{N}}=\frac{A_{N}(1-n)^{1-\beta}}{z^{1-\beta}}-\frac{(1-\gamma)}{p_{N}} \frac{p_{C} C}{K_{N}}
$$


Usando la ecuación (24), el valor de $p_{C} C / K_{N}$ es:

$$
\frac{p_{C} C}{K_{N}}=\frac{\hat{c}}{z}\left[A_{T} n^{1-\alpha}+p_{N} A_{N} z^{\beta}(1-n)^{1-\beta}-\left(r^{*}+\xi\right) d\right] .
$$

Asimismo, es posible mostrar que la tasa de crecimiento del producto total es:

$$
\begin{aligned}
\frac{\dot{Y}}{Y} & =\frac{Y_{T}}{Y}\left[\frac{\dot{K_{T}}}{K_{T}}+(1-\alpha) \frac{\dot{n}}{n}\right] \\
& +\frac{p_{N} Y_{N}}{Y}\left[\beta \frac{\dot{\mathrm{Z}}}{\mathrm{z}}+\frac{\dot{K_{T}}}{K_{T}}-(1-\beta) \frac{\dot{n}}{n} \frac{n}{(1-n)}+\frac{\dot{p_{N}}}{p_{N}}\right]
\end{aligned}
$$

donde $Y_{T} / Y=A_{T} n^{1-\alpha} /\left[A_{T} n^{1-\alpha}+p_{N} A_{N} z^{\beta}(1-n)^{1-\beta}\right]$ es la participación de $Y_{T}$ en el producto total y $p_{N} Y_{N} / Y=p_{N} A_{N} z^{\beta}(1-n)^{1-\beta} /\left[\left(A_{T} n^{1-\alpha}+p_{N} A_{N} z^{\beta}(1-n)^{1-\beta}\right]\right.$ es la participación de $p_{N} Y_{N}$ en el producto total. En la siguiente sección se muestra el sistema dinámico de la economía.

\section{EL SISTEMA DINÁMICO}

Una vez redefinidas las ecuaciones del modelo, es posible obtener un sistema de ecuaciones diferenciales para esta economía. Como será evidente en la sección IV, el valor de $n$ siempre se encuentra en un estado estacionario. Con esto es posible definir el sistema dinámico respecto a dos variables estacionarias, $d$ y $p_{N}$. El modelo dinámico es:

$$
\begin{aligned}
& \dot{d}=f_{1}\left(d, p_{N}\right) \\
& \dot{p}_{N}=f_{2}\left(d, p_{N}\right)
\end{aligned}
$$

en donde $f_{1}$ y $f_{2}$ son funciones no lineales. Una vez determinadas estas variables se determinan las restantes.

A continuación se obtiene el modelo dinámico. Tomando logaritmos y derivadas respecto al tiempo de $z=K_{N} / K_{T}$, se obtiene:

$$
\frac{\dot{z}}{z}=\frac{\dot{K_{N}}}{K_{N}}-\frac{\dot{K_{T}}}{K_{T}} .
$$


Con la ecuación (22), se obtiene:

$$
z=\left[\frac{A_{T}(1-\alpha)(1-n)^{\beta}}{p_{N} A_{N}(1-\beta) n^{\alpha}}\right]^{\frac{1}{\beta}} .
$$

Considerando que $n$ siempre se encuentra en un estado estacionario y tomando logaritmos y derivadas respecto al tiempo de la ecuación (31), se obtiene el valor de $\dot{z} / z$ :

$$
\frac{\dot{z}}{z}=-\frac{1}{\beta} \frac{\dot{p_{N}}}{p_{N}}
$$

Utilizando las ecuaciones (30) y (32), se obtiene:

$$
\frac{\dot{K}_{T}}{K_{T}}=\frac{\dot{K}_{N}}{K_{N}}+\frac{1}{\beta} \frac{\dot{p}_{N}}{p_{N}}
$$

Por tanto, la primera ecuación diferencial del sistema está definida por la ecuación (23) con las ecuaciones (21), (25), (26), (27), (31) y (33). La segunda ecuación diferencial del sistema está definida por las ecuaciones (21) y (31). En la sección IV se muestra la solución en el estado estacionario y cómo la economía responde a una mayor movilidad del capital externo.

\section{LA SOLUCIÓN DE ESTADO ESTACIONARIO Y LA DINÁMICA DE TRANSICIÓN}

La solución de estado estacionario implica la existencia del equilibrio. Así, dado que los valores de $z, n, d$ y $p_{N}$ son constantes en el estado estacionario, las tasas de crecimiento de $z, n, d$ y $p_{N}$ son igual a 0 en el estado estacionario. Asimismo, dado que las tasas de crecimiento de $K_{T}, K_{N}, Y_{T}, Y_{N}$ y $Y$ dependen solamente de $z, n, d, p_{N}$ y parámetros, es posible afirmar que sus tasas de crecimiento son constantes en el estado estacionario y que crecen a una misma tasa $g^{*}$ de estado estacionario (se denota a los valores de estado estacionario con un asterisco). Por tanto, de la ecuación (18) se tiene que:

$$
n^{*}=\left(\frac{r^{*}+\xi}{A_{T} \alpha}\right)^{\frac{1}{1-\alpha}}
$$


Como una mayor movilidad del capital es cuando $\xi$ disminuye, se tiene que $\partial n^{*} / \partial \xi>0$. Por tanto, el valor de $n^{*}$ disminuye en el nuevo estado estacionario. Dado que no existe un costo en la movilidad del trabajo, el nivel de $n^{*}$ brinca a su nuevo valor de estado estacionario. La condición de equilibrio dinámica para $K_{N}$, ecuación (21), se puede reescribir como:

$$
\frac{\dot{p}_{N}}{p_{N}}=\left(r^{*}+\xi\right)-A_{N} \beta \mathrm{z}^{\beta-1}(1-n)^{1-\beta} .
$$

Sustituyendo el valor de $z$, ecuación (31), en la ecuación (35), se obtiene:

$$
\frac{\dot{p}_{N}}{p_{N}}=\left(r^{*}+\xi\right)-A_{N} \beta\left[\frac{A_{T}(1-\alpha)(1-n)^{\beta}}{p_{N} A_{N}(1-\beta) n^{\alpha}}\right]^{\frac{\beta-1}{\beta}}(1-n)^{1-\beta} .
$$

Dado que $n^{*}$ es una constante y que $p_{N}$ es constante en el estado estacionario, de la ecuación (36) se tiene que:

$$
r^{*}+\xi=A_{N} \beta\left(1-n^{*}\right)^{1-\beta}\left[\frac{p_{N} A_{N}(1-\beta) n^{* \alpha}}{A_{T}(1-\alpha)\left(1-n^{*}\right)^{\beta}}\right]^{(1-\beta) / \beta} .
$$

Despejando $p_{N}$ de la ecuación anterior, se obtiene:

$$
p_{N}=\left[\frac{1}{\left(A_{N} \beta\right)^{\beta /(1-\beta)}}\right]\left[\frac{A_{T}(1-\alpha)}{A_{N}(1-\beta)}\right]\left[\frac{\left(r^{*}+\xi\right)^{\beta /(1-\beta)}}{n^{* \alpha}}\right] .
$$

Finalmente, sustituyendo el valor de $n^{*}$, se obtiene el valor $p_{N}$ en el estado estacionario:

$$
p_{N}^{*}=\left[\frac{\left(A_{T} \alpha\right)^{\alpha /(1-\alpha)}}{\left(A_{N} \beta\right)^{\beta /(1-\beta)}}\right]\left[\frac{A_{T}(1-\alpha)}{A_{N}(1-\beta)}\right]\left[\left(r^{*}+\xi\right)^{[(\beta-\alpha) /(1-\beta)(1-\alpha)]}\right] .
$$

Asimismo, dado que $\alpha>\beta$, se puede demostrar que $\partial p_{N}^{*} / \partial \xi<0$. Así, se tiene que una mayor movilidad del capital conducirá a un aumento del capital externo y a una apreciación del tipo de cambio real (aumento de $p_{N}^{*}$ ).

Sustituyendo la ecuación (39) en (31), se obtiene el valor de $z$ en el estado estacionario: 


$$
z^{*}=H_{2}\left[\frac{\left(r^{*}+\xi\right)^{-[(1-\alpha)+\alpha(\alpha-\beta)]}}{H_{1} H_{3}}\right]\left[1-H_{3}\left(r^{*}+\xi\right)^{1 /(1-\alpha)}\right],
$$

en donde $H_{1}, H_{2}$ y $H_{3}$ son parámetros:

$$
\begin{gathered}
H_{1}=\left[\frac{\left(A_{T} \alpha\right)^{\alpha /(1-\alpha)}}{\left(A_{N} \beta\right)^{\beta /(1-\beta)}}\right]\left[\frac{A_{T}(1-\alpha)}{A_{N}(1-\beta)}\right] \\
H_{2}=\left[\frac{A_{T}(1-\alpha)}{A_{N}(1-\beta)}\right]^{1 / \beta} \\
H_{3}=\left(\frac{1}{A_{T} \alpha}\right)^{1 /(1-\alpha)} .
\end{gathered}
$$

Se puede demostrar que $\partial z^{*} / \partial \xi<0$; así, una mayor movilidad del capital externo conduce a un aumento en el valor de $z=K_{N} / K_{T}$.

Resumiendo, un aumento en la movilidad del capital conduce a una entrada de capital externo y a una apreciación del tipo de cambio real (aumento en $p_{N}{ }_{N}$ ). Esta apreciación estimula la acumulación de capital en el sector no comerciable (no manufacturero) y desalienta la acumulación de capital en el sector comerciable (manufacturero), dando por resultado un aumento de $z^{*}$. Asimismo, la fracción del trabajo empleado en el sector comerciable disminuye. Por tanto, una mayor movilidad del capital siempre daña al sector comerciable.

A continuación se obtienen los valores de $d$ y $g$ de estado estacionario. Por tanto, se tiene que $\dot{d}=0$ y $\dot{K}_{T} / K_{T}=g$; con las ecuaciones (23) y (25) se obtiene:

$$
\begin{gathered}
g^{*}=\left[\frac{1}{1-d}\right]\left[A_{T} n^{1-\alpha}-\gamma \hat{c}\left[A_{T} n^{1-\alpha}+p_{N} A_{N} z^{\beta}(1-n)^{1-\beta}\right]\right. \\
\left.+\left(r^{*}+\xi\right) d(\gamma \hat{c}-1)\right] .
\end{gathered}
$$

Del mismo modo, en el estado estacionario se tiene que $\dot{K}_{N} / K_{N}=g^{*}$. Usando las ecuación (26) y (27), se obtiene:

$$
g^{*}=\frac{A_{N}(1-n)^{1-\beta}}{z^{1-\beta}}-\frac{(1-\gamma) \hat{c}}{p_{N} z}\left[A_{T} n^{1-\alpha}+p_{N} A_{N} z^{\beta}(1-n)^{1-\beta}\right]+\frac{(1-\gamma) \hat{c}\left(r^{*}+\xi\right) d}{p_{N} z}
$$


Igualando las ecuaciones (41) y (42) y utilizando $p_{N}^{*}$, se obtiene un polinomio en $d$ de estado estacionario:

$$
H_{4} d^{2}+H_{5} d+H_{6}=0
$$

en donde $H_{4}, H_{5}$ y $H_{6}$ son los coeficientes del polinomio:

$$
\begin{gathered}
H_{4}=\frac{(1-\gamma)}{p_{N}^{*}} \frac{\hat{c}}{z^{*}}\left(r^{*}+\xi\right) \\
H_{5}=H_{7}+\left(r^{*}+\xi\right)(\gamma \hat{c}-1)-\frac{(1-\gamma)}{p_{N}^{*}} \frac{\hat{c}}{z^{*}}\left(r^{*}+\xi\right) \\
H_{6}=A_{T} n^{* 1-\alpha}-\gamma \hat{c}\left[A_{T} n^{* 1-\alpha}+p_{N}^{*} A_{N} z^{* \beta}\left(1-n^{*}\right)^{1-\beta}\right]-H_{7} \\
H_{7}=\left[\frac{A_{N}\left(1-n^{*}\right)^{1-\beta}}{z^{* 1-\beta}}-\frac{(1-\gamma)}{p_{N}^{*}} \frac{\hat{c}}{z^{*}}\left[A_{T} n^{* 1-\alpha}+p_{N}^{*} A_{N} z^{* \beta}\left(1-n^{*}\right)^{1-\beta}\right]\right] .
\end{gathered}
$$

La solución para $d$ está dada por las raíces del polinomio, $d_{1}^{*}, d_{2}^{*}=$ $\left[-H_{5} \pm\left(H_{5}^{2}-4 H_{4} H_{6}\right)^{1 / 2}\right] / 2 H_{4}$. Como fue imposible conocer analíticamente los signos de los coeficientes, se realizaron simulaciones numéricas para determinar los valores $d_{1}^{*}$ y $d_{2}^{*}$. En este artículo, se consideran los casos cuando $d$ es positivo y se desechan los casos de cambio de régimen, es decir, cuando el individuo cambia de deudor a prestatario (cuando $d$ es negativa habría que cambiar el significado de $\xi$ y del modelo). Así, se encontraron tanto soluciones en donde hay un solo valor positivo para $d^{*}$, como soluciones en donde existen dos valores positivos para $d^{*}$ (equilibrio múltiple). También es posible no encontrar soluciones. Esto es producto de los rendimientos crecientes a escala. Por tanto, una mayor movilidad del capital puede conducir a diferentes niveles de endeudamiento y a una mayor o menor tasa de crecimiento en el largo plazo.

Es importante destacar que se comprobó analíticamente que las variables $n^{*}, \mathrm{z}^{*}$ y $p_{N}^{*}$ tienen un solo valor de equilibrio y que una mayor movilidad del capital siempre daña al sector comerciable por medio de un aumento en $p_{N}^{*}$, disminuyendo $n^{*}$ y aumentando $z^{*}$. Mientras que $d^{*}$ y $g^{*}$ pueden tener cero, uno o dos valores de equilibrio.

Para entender la relación entre mayor movilidad del capital, mayor flujo de capital externo y crecimiento en el estado estacionario, se presenta una simu- 
lación representativa en donde el parámetro $\xi$ es disminuido hasta cero. Los valores de los parámetros son: $\alpha=0.3, \beta=0.2, \gamma=0.3, \hat{c}=0.9, A_{T}=0.4, A_{N}=0.4$ y $r^{*}=0.03$. Estos valores son sólo para propósitos ilustrativos. Primero, se considera que $\xi=0.02$, es decir, la tasa de interés sobre los activos domésticos y la deuda externa se ubica a 200 puntos base sobre la tasa de interés mundial. El resultado numérico es: $p^{*}{ }_{N}=1.1322, n^{*}=0.2863$ y $z^{*}=1.2843$. Para $d^{*}$ la economía muestra un equilibrio múltiple. La economía puede estar situada en un nivel alto o bajo de $d^{*}$. En el equilibrio alto, el nivel de $d^{*}$ es 1.6585 y le corresponde una $g^{*}$ de $5.62 \%$ por año. En el equilibrio bajo, el nivel de $d^{*}$ es 0.0899 y $g^{*}=$ $2.22 \%$ por año.

Ahora se considera que $\xi$ disminuye a 0.01 , es decir, la tasa de interés sobre los activos domésticos y la deuda externa se ubica a 100 puntos base sobre la tasa de interés internacional. Esta disminución de $\xi$ implica una mayor movilidad del capital y una entrada de capital externo a la economía. El resultado numérico es: $n^{*}=0.2082, z^{*}=1.8833$ y $p^{*}{ }_{N}=1.1782$. Como se observa, las variables $z^{*}, n^{*}$ y $p^{*}{ }_{N}$ se mueven en la dirección predicha analíticamente. Por tanto, el precio relativo del bien no comerciable aumenta, es decir, se produce una apreciación del tipo de cambio real, resultando que el sector no comerciable atraiga recursos a costa del sector comerciable; así, el nivel de $n^{*}$ disminuye y $z^{*}$ aumenta. El sector no comerciable emplea más trabajo y acumula relativamente más capital que el sector comerciable. Por tanto, como siempre en este modelo, un aumento en la movilidad del capital daña al sector comerciable y esto puede perjudicar o beneficiar la tasa de crecimiento de la economía. Para $d^{*}$ la economía muestra un solo equilibrio positivo. Por tanto, el nivel de $d^{*}$ aumenta de 1.6585 a 2.4748 o de 0.0899 a 2.4748 . En ambos casos la deuda relativa a $K_{T}$ aumenta, por la mayor movilidad del capital. Asimismo, la tasa de crecimiento de la economía pasa de 5.62 a 6.43 o de 2.22 a $6.43 \%$ anual. Para ambos casos la tasa de crecimiento aumenta. Así, para este caso, el flujo de capital externo beneficia el crecimiento económico.

Ahora se considera que $\xi$ es igual a 0 , por lo que la tasa de interés sobre los activos domésticos y la deuda externa es igual a la tasa de interés mundial. El resultado numérico es: $p^{*}{ }_{N}=1.2403, n^{*}=0.1380 \mathrm{y} z^{*}=2.9374$. Nuevamente, las variables $z^{*}, n^{*}$ y $p_{N}^{*}$ se mueven en la dirección predicha analíticamente. La entrada de capital externo produce un aumento de $p^{*}{ }_{N}$ y una apreciación del tipo de cambio real. Así, $n^{*}$ disminuye y $z^{*}$ aumenta, es decir, el sector comerciable es perjudicado. La economía tiene un solo equilibrio para $d^{*}$. El nivel de $d^{*}$ aumenta de 2.4748 a 3.7121 . Ahora, la tasa de crecimiento disminuye de 6.43 a $5.75 \%$ 
anual. En este caso, el flujo de capital externo perjudica el crecimiento económico. Resumiendo, la entrada de capital externo puede beneficiar o dañar el crecimiento. Esta relación ambigua entre movilidad de capital y crecimiento económico da apoyo a la bibliografía empírica.

Ahora se explica la dinámica de transición. Primero, se linealizó el sistema dinámico (29) alrededor de un punto de estado estacionario. Utilizando los mismos valores de los parámetros, se calcularon los valores característicos del sistema linealizado y se obtuvo una raíz positiva y una negativa. Por tanto, la dinámica del modelo es de punto silla. Así, existe una variable que se mueve lentamente y otra que brinca. Por definición, la variable $d$ se mueve lentamente, como la acumulación de deuda externa-capital comerciable es pausada. Dado que en este modelo hay plena flexibilidad del precio relativo del bien no comerciable, el nivel de $p_{N}$ sí puede brincar. Por tanto, se puede afirmar que la variable $d$ es una variable de movimiento lento y la variable $p_{N}$ es una variable que brinca en el momento 0 .

\section{CONCLUSIONES}

Se desarrolló un modelo de crecimiento endógeno con dos sectores productivos, en donde el sector comerciable es el único que genera progreso técnico. El conocimiento generado en el sector comerciable se utiliza en el sector no comerciable. Estos dos sectores tienen la capacidad de acumular capital. Se asumió que el gasto total en consumo depende del ingreso total disponible. Las demandas para el bien comerciable y no comerciable se obtuvieron por medio de la maximización de una función de utilidad. Es posible identificar al sector comerciable como el sector manufacturero y al sector no comerciable como el sector no manufacturero (construcción y servicios). Con el modelo, se estudió la relación entre movilidad de capital, tipo de cambio real y crecimiento económico.

Se demostró analíticamente que una mayor movilidad del capital produce una apreciación del tipo de cambio real. Esta apreciación ocasiona que la fracción de trabajo empleado en el sector comerciable disminuya. Asimismo, la acumulación de capital en el sector comerciable se desalienta y la acumulación de capital en el sector no comerciable se estimula, dando por resultado un aumento en la relación de capitales. Por tanto, el modelo siempre predice que la liberalización financiera daña al sector comerciable que genera el progreso técnico. Sin embargo, dado que existen rendimientos crecientes a escala, se mostró numéricamente que la relación deuda-capital-comerciable aumenta y que la tasa de 
crecimiento de la economía puede disminuir o aumentar en el largo plazo. Así, el resultado ambiguo entre movilidad de capital y crecimiento económico apoya la bibliografía empírica, en donde la gran mayoría de los estudios macroeconómicos muestran una no relación entre integración financiera internacional y crecimiento. Por ello, dependiendo de las características de cada economía y principalmente de las intensidades de los rendimientos crecientes a escala, un aumento en la movilidad del capital puede dañar o beneficiar el crecimiento de una economía.

\section{REFERENCIAS BIBLIOGRÁFICAS}

Aizenman, J., B. Pinto, y A. Radziwill (2007), "Sources for financing domestic capital-is foreign saving a viable option for developing countries?", Journal of International Money and Finance, Vol. 26, pp. 682-702.

Barro, R. J., y X. Sala-i-Martin (1995), Economic Growth, McGraw-Hill.

Brock, P. L., y S. J. Turnovsky (1994), “The Dependent-Economy Model with Both Traded and Nontraded Capital Goods", Review of International Economics, Vol. 2, pp. 306-325.

Eicher, T., y L. Hull (2004), "Financial Liberalization, Openness and Convergence", Journal of International Trade \& Economic Development, Vol. 13, páginas 443-459.

— y S. J. Turnovsky (1999), "International Capital Markets and Non-Scale Growth”, Review of International Economics, Vol. 7, pp. 171-188.

Gala, P. (2008), "Real exchange rate levels and economic development: Theoretical analysis and econometric evidence", Cambridge Journal of Economics, Vol. 32, pp. 273-288.

Henry, P. B. (2003), "Capital-Account Liberalization, the Cost of Capital, and Economic Growth”, AEA Papers and Proceedings, Vol. 93, núm. 2, pp. 91-96.

Kose, M. A., E. Prasad, K. Rogoff, y S. J. Wei (2006), "Financial Globalization: A Reappraisal", NBER Working Paper, núm. 12484.

Neto, D. G. (2009), "Financial Globalization and Economic Growth", Universidad de Vigo.

Obstfeld, M. (1989), "Fiscal Deficits and Relative Prices in a Growing World Economy", Journal of Monetary Economics, Vol. 23, pp. 461-484.

(2008), "International Finance and Growth in Developing Countries: What Have We Learned?", Documento de Trabajo 34, Comisión sobre Crecimiento y Desarrollo. 
Prasad, E., R. G. Rajan, y A. Subramanian (2006), "Patterns of International Capital Flows and Their Implications for Economic Development", en Symposium Proceedings The New Economic Geography: Effects and Policy Implications, Reserva Federal, Bank of Kansas City.

$\longrightarrow$ - - - - (2007), "Foreign Capital and Economic Growth", Brookings Papers on Economic Activity, núm. 1, pp. 153-230.

Rodrik, D. (2008), “The Real Exchange Rate and Economic Growth”, Brookings Papers on Economic Activity, Vol. 2, pp. 365-412.

Romer, P. M. (1989), "Capital Accumulation in the Theory of Long Run Growth", en Barro (editor), Modern Business Cycle Theory, Basil Blackwell.

Torvik, R. (2001), "Learning by Doing and the Dutch Disease", European Economic Review, Vol. 45, pp. 285-306.

Turnovsky, S. J. (1996), "Endogenous Growth in a Dependent Economy with Traded and Nontraded Capital”, Review of International Economics, Vol. 4, pp. 300-321.

(1997), International Macroeconomic Dynamic, MIT Press.

Urrutia, C., y F. Meza (2010), "Financial Liberalization, Structural Change, and Real Exchange Rate Appreciations”, IMF Working Paper, núm. 63. 